\title{
Mortality rate after stepwise coronary occlusion when adhesion to the ischaemic myocardium is prevented ${ }^{1}$
}

\author{
PANAGIOTISC. PETROPOULOS ${ }^{2}$
}

From the Department of Cardiovascular Surgery, the Presbyterian Medical Center, San Francisco, Calif.

It is well known that the mortality rate after a standard gradual occlusion of the upper segment of the anterior descending coronary artery varies in the literature from 0 to $100 \%$. This variation is attributed to the following: the level and extent of occlusion of the coronary artery ; the origin of the septal artery; the existence of branches of the circumflex coronary artery vascularizing the angle between this artery and the anterior descending coronary artery; the age, race, and condition of the dogs; the type of anaesthesia and ventilation used during the operation ; and the surgeon's technique. Although, after occlusion of a main coronary artery, spontaneous adhesions of the surrounding tissues are formed to the ischaemic myocardium, through which extracoronary vascular anastomotic channels are built, there is no information in the literature regarding the mortality rate when the formation of such adhesions is prevented. In order to study this the following experiments have been performed.

\section{METHODS}

Sixty unselected healthy mongrel dogs were used, whose weights ranged from 14 to $21 \mathrm{~kg}$. Anaesthesia was induced and continued with intravenous nembutal, $30 \mathrm{mg}$. per $\mathrm{kg}$. body weight. Cyclic positive pressure insufflation of the lungs was maintained with a mixture of $50 \%$ oxygen and air, and the thorax was opened through the left fourth interspace. The pericardium was incised and the left coronary artery was identified.

It is important at this stage to note the coronary artery variations. The branches of the left anterior

1 Supported by grants from the American Heart Association and the U.S. Public Health Service

2 Fellow in Cardiovascular Surgery, Cora and Webb Mading Departm:nt of Surgery, Baylor University College of Medicine, Houston, Texas, and Stanford University Medical School, Palo Alto, California, and the Institute of Medical Sciences, Presbyterian Medical Center, San Francisco, California descending coronary artery were distributed predominantly in the left ventricle in 42 dogs (type $A)_{3}$ and in both left and right ventricles in 18 dogs (type B) (Lochner and Witzled, 1938). The angle between $\widehat{\widehat{C}}$ the circumflex and anterior descending coronary arteries was vascularized from a branch arising from\& the circumflex artery in 39 dogs and from the anterior $\AA$ descending coronary artery in 21 dogs. The anterior descending coronary artery was then freed for 3 to $5 \mathrm{~cm}$. up to the origin of the circumflex coronary artery. The origin of the septal artery (Moore, 1930) was from the main branch of the left coronary arteryo in 26 dogs and from the first $3 \mathrm{~mm}$. of the anterior descending coronary artery in the remaining 34 dogs $\overrightarrow{\vec{A}}$ (Fig. 1). The anterior descending coronary artery was 3 occluded temporarily just distal to the septal artery, and peripheral anterior descending coronary artery pressure was determined using a No. 18-21 gauge needle connected to a polyethylene catheter. The $\overrightarrow{\vec{b}}$ catheter was connected to a strain gauge, and readings were taken on a Sanborn polywiso recorder. Peripheral $\times$ coronary artery pressure readings were recorded about 15 seconds after occlusion of the artery.

In order to obtain a standard segmental thrombosis over some length of the vessel, a 2 to $2.5 \mathrm{~cm}$. long magnesium-aluminium-alloy wire (Gage, Olson, ando Chardack, 1956) with a diameter of 1.0 to $1.5 \mathrm{~mm}$.? depending on the diameter of the coronary artery $\frac{D}{0}$ was completely inserted in the lumen of the artery?. $3 \mathrm{~mm}$. below the level of the septal artery. The N haemorrhage which resulted from the puncture wound was controlled with a drop of plastic adhesive (2- $N$ cyanoacrylate).

In the first series of 30 dogs the pericardium waso approximated with three sutures and the chest waso closed in a routine manner. In a second series of 15 ? dogs the pericardium was left widely open in order( to create optimum conditions for the formation of adhesions. In a third series of 15 dogs the expected pericardial and pulmonary adhesions to the ischaemic ventricular myocardium were prevented by isolating the anterior surface of the left ventricle with a thin $\mathbb{\Omega}^{\circ}$ nylon sheet $0.5 \mathrm{~mm}$. thick fixed to the internal surface of the anterior pericardium (Fig. 2). The pericardium 


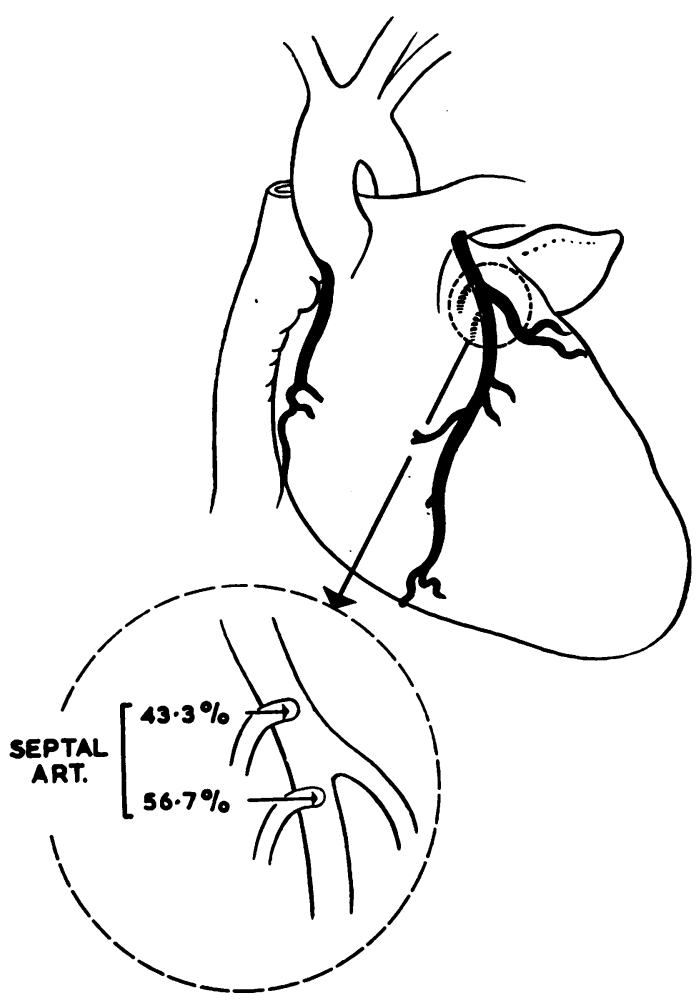

FIG. 1. Origin of the septal artery.

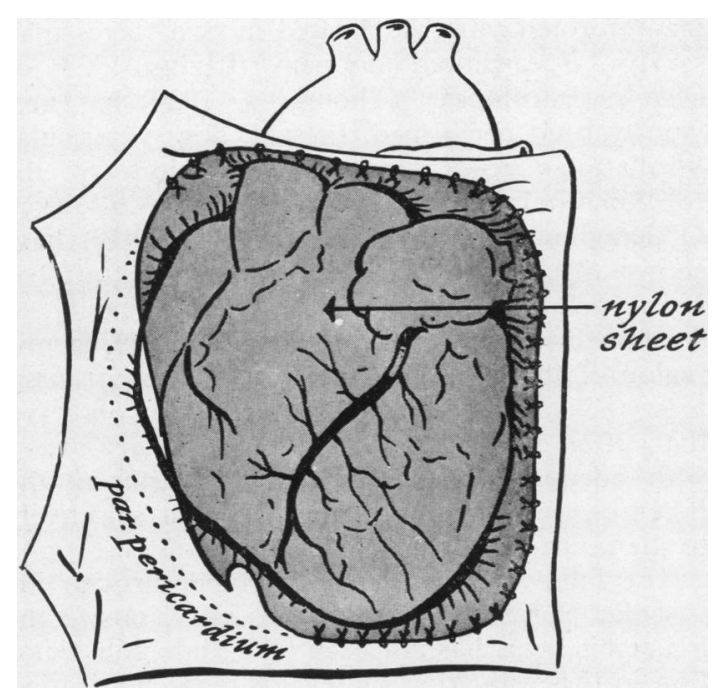

FIG. 2. A nylon sheet isolates the anterior surface of the ischaemic left ventricle. was then approximated with two sutures and the chest was closed.

In five supplementary control dogs with an anterior descending coronary artery distribution of type $A$, a similar nylon sheet was transposed and fixed between the visceral and parietal pericardium in such a way that any compression of the underlying coronary arteries would be avoided.

\section{RESULTS}

Of the 30 dogs in the first group 20 died during the first 11 post-operative days $(66.6 \%$ mortality). Myocardial transmural infarction was present in 11 animals and subendocardial infarction in the remaining nine. The segment of the anterior coronary artery in which the wire had been inserted was thrombosed in all the specimens. In four specimens the septal artery was also thrombosed. The surviving animals were killed 32 to 60 days after insertion of the wire. Before sacrificing each animal, the retropressure of the still patent distal segment of the artery was recorded. These pressures equalled or exceeded the previous prethrombotic pressures and were between 40/32 and $76 / 43 \mathrm{~mm}$. $\mathrm{Hg}$. The backflow was between 1.0 and $2.1 \mathrm{ml}$. per minute. Light pericardial adhesions were found to the anterior surface of the left ventricle. When the adhesions were separated some bleeding occurred. On section a thrombosis of the septal artery was present in one animal. In one surviving dog occlusion of the anterior descending coronary artery was not complete. When the artery was examined, complete disintegration of the wire was noted. Evidence of healed infarction of the anterior left ventricular wall was present in six animals.

Of the 15 dogs in the second group, eight died during the first 13 post-operative days $(53.3 \%$ mortality). Myocardial transmural infarction was found in three specimens and subendocardial infarction in five. The extent of the infarction was about the same as in the previous group. The septal artery was patent. The coronary segment with the metallic implant was occluded in all the specimens. The surviving dogs were operated on a second time and killed 40 to 60 days postoperatively. At re-operation extensive adhesions between the left lung and the left ventricular myocardium were found. After separation of the adhesions the bleeding was more extensive and persistent than in the previous group. The retropressures distally to the occluded artery, before separation of the adhesions, were between $44 / 35$ and $74 / 42 \mathrm{~mm}$. $\mathrm{Hg}$. The backflow was between 
1.3 and $2.5 \mathrm{ml}$. per minute. Healed subendocardial infarction was found on section.

All 15 dogs in the third group died during the first eight post-operative days. Infarction of the anterior surface of the left ventricle was present, being subendocardial in six dogs and transmural in nine. Segmental occlusion of the anterior descending coronary artery was evident in all the specimens. The myocardial transmural infarction was 20 to $40 \%$ greater than in the other two groups. An infarction of the septum was also present in three animals.

The extent of anastomosis between the cardiac and extracardiac vessels was studied in the hearts of nine dead dogs, three from each group. The heart was excised within 30 minutes of death. The aorta was split longitudinally, and a cannula was inserted and fixed into the left coronary ostium. The injection material used was a $30 \%$ suspension of lamp black and $5 \%$ acacia in distilled water, warmed to a temperature of $40^{\circ} \mathrm{C}$. (Hudson, Moritz, and Wearn, 1932). Before injection, suction was applied for a few minutes while the heart was massaged in order to withdraw air and blood from the vessel. The specimens were then injected in a water bath at the same temperature and massaged during injection. The dye was injected under positive pressure of about $200 \mathrm{mg}$. of mercury for 10 minutes. The entire specimen was then placed in $10 \%$ formalin for 24 hours. Blocks were cut and sections prepared to study the distribution of the material in the myocardium and pericardium. In the first group of animals, in which adhesions to the infarcted area were present, carbon particles were present in the infarcted area and in the adjacent pericardium. In the second group, the carbon particles were found in the infarcted area and in the adherent left lung. In the third group, when a nylon sheet was placed between the left myocardium and the parietal pericardium, the carbon particles penetrated only to the borders of the infarcted area but not to the corresponding parietal pericardium.

All five control dogs survived till the predicted re-operation and sacrifice time, which was 100 days after the initial transposition of the nylon sheet. At re-operation a fibrosanguineous exudate of the pleura was present in all the animals. The nylon sheet was strongly embedded between extensive scar formations of the lung and pericardium. The retropressures of the anterior descending coronary artery were between 50/39 and $72 / 43$ $\mathrm{mm}$. $\mathrm{Hg}$. The backflow was between $1 \cdot 2$ and $2 \cdot 1$ ml. per minute.

\section{DISCUSSION}

From the above. experiments it was found that, $\underline{\underline{C}}$ when spontaneous adhesions to the ischaemic $\overline{\bar{s}}$. myocardium from the adjacent parietal peri- $\frac{\Phi}{0}$ cardium or lung were formed, the mortality rates were 66.6 and $53.3 \%$ respectively. On the other hand, when the adhesions were prevented, the $\vec{\circ}$ mortality rate was $100 \%$. The simple pericardial $\overrightarrow{\vec{\omega}}$ transposition of a nylon sheet caused no deaths, $\stackrel{\omega}{\sigma}$ although the reaction of the surrounding tissues $\vec{x}$ was strong.

These findings suggest that, in addition to the known causes of mortality after gradual coronary $\vec{\sigma}$ occlusion, one more factor must be considered. $\sigma$ That is the extent of the vascular adhesions of the surrounding tissues to the infarcted area of the heart.

It is thought that after gradual occlusion of a coronary artery the compensating vascular supply $\overrightarrow{0}$ is made possible through development of (1) intracoronary communications and (2) extracoronary ${ }^{+}$ anastomosis (Gregg and Sabiston, 1956). From the results of our experiments it can be suggested that when the development of extracoronary ana-o stomosis is completely prevented the evolution of $\stackrel{2}{\circ}$ the intracoronary anastomosis alone may not be@ sufficient to ensure the viability of the heart.

The ability of the ischaemic myocardium to survive when supplied only with blood arising from extracardiac sources through anastomosis is well known. It has been shown that dogs with both main coronary arteries ligated in two stages may survive, if cardiopulmonopexy (Lezius, 1938) or even pericardiopexy (Thompson, 1939) has pre-3. viously been performed. In such dogs ventricular fibrillation occurs when the adhesions of the cardiopulmonopexy are divided. In addition, O'Shaughnessy, Slome, and Watson (1939) have? shown that, when a pedicled omentum graft has been loosely fixed in the pericardial cavity, it spontaneously becomes adherent to the ischaemic N region of the myocardium after a main coronary artery is ligated. We have also repeatedly observed that, after obstruction of a large coronary artery ${ }_{\sigma}^{\omega}$ with adequate closure of the pericardium, the latter has been found to be adherent selectivelyo to the infarcted region of the myocardium.

The inflow of extracardiac blood through the vascular bed of the spontaneous adhesions to the ischaemic heart has not been taken into considera- $\frac{\vec{D}}{\mathrm{D}}$ tion in the evaluation of several revascularization procedures of the heart. In some previous experi离 ments, for example, after implantation of the 
internal mammary artery in the ischaemic left ventricle, we obtained a mortality rate of $67 \%$ when spontaneous adhesions to the ischaemic myocardium were formed and $100 \%$ when adhesions were prevented from forming by the insertion of a nylon sheet (Petropoulos, 1963a). In some other unsuccessful attempts to revascularize the left ischaemic ventricle from the left atrium, the mortality rate was 57 to $70 \%$ when adhesions were not prevented from forming and $100 \%$ when the formation of adhesions was prevented by a nylon sheet (Petropoulos, 1963b).

It could be suggested that, by preventing spontaneous adhesions of the pericardium and lung to the ischaemic myocardium, paradoxical dilatation of the ischaemic non-contracted region during cardiac systole would not be restricted. This would decrease the cardiac output and contribute to the high mortality rate. By partially suturing the pericardium over the interposed nylon sheet we did not exclude the protective action of the pericardium on the expected dilatation of the infarcted myocardial area during ventricular systole.

\section{SUMMARY}

Gradual occlusion of a $2 \mathrm{~cm}$. segment of the anterior descending coronary artery distal to the origin of the septal artery has given a mortality of $66.6 \%$ in a series of 30 control dogs in which the pericardium was approximated and $53.3 \%$ in a second series of 15 dogs in which the pericardium was left widely open. When a nylon sheet was interposed between the infarcted area and the parietal pericardium, the mortality was $100 \%$. Special attention is drawn to the effect on mortality rate of spontaneous vascular adhesions.

\section{REFERENCES}

Gage, A. A., Olson, K. C., and Chardack, W. M. (1956). Experimental coronary thrombosis in the dog. Description of a method. Ann. Surg., 143, 535.

Gregg, D. E., and Sabiston, D. C. (1956). Current research and problems of the coronary circulation. Circulation, 13, 916.

Hudson, C. L., Moritz, A. R., and Wearn, J. T. (1932). The extracardiac anastomoses of the coronary arteries. J. exp. Med. 56, 919 .

Lezius, A. (1938). Die anatomische und functionellen Grundlagen der künstlichen Blutversorgung der Herzmuskels durch die der kunstlichen Blutversorgung der Herzmuskels durch die

Lochner, W. and Witzled, E. (1938). Probleme der Coronardurchblutung. Springer, Göttingen, Berlin.

Moore, R. A. (1930). The coronary arteries of the dog. Amer. Heart J., 5, 743.

O'Shaughnessy, L., Slome, D., and Watson, F. (1939). Surgical revascularization of the heart : the experimental basis. Lancet, 1,617 .

Petropoulos, P. C. (1963a). Ability of the ischaemic myccardium to absorb blood perfused artificially through a previously implanted artery. Thorax, 18, 251 .

( $1963 \mathrm{~b})$ Fate of some revascularization procedures of the heart, providing a constant contact between saturated blood at low pressures and an area of the ischaemic left ventricular myocardium. J. cardiovasc. Surg. (Torino), 4, 769 .

Thompson, S. A. (1939). Development of cardio-pericardial adhesions following the use of talc. Proc. Soc. exp. Biol., N.Y., 40, 260. 